\title{
Theory of photoluminescence in quantum wells in the presence of transverse electric field: Monte Carlo approach
}

\author{
Jasprit Singh \\ Department of Electrical and Computer Engineering, The University of Michigan, \\ Ann Arbor, Michigan 48109
}

(Received 14 October 1985; accepted for publication 6 January 1986)

\begin{abstract}
The Monte Carlo approach is used to study the quasibound electron and hole ground-state levels in a quantum well in the presence of a transverse electric field. A criteria is developed to ensure that the ground state is a true quasibound state. These techniques are applied to an $\mathrm{Al}_{0.3} \mathrm{Ga}_{0.7} \mathrm{As} /$ $\mathrm{GaAs}$ quantum well subjected to a high electric field perpendicular to the interfaces. Results on the electron and hole tunneling rates and their dependence on band-edge discontinuities are presented. The variation of the ground-state emission energy with the electric field is also presented.
\end{abstract}

The advances in epitaxial crystal growth techniques such as molecular-beam epitaxy (MBE) and metalorganic chemical vapor deposition have led to a number of novel proposals for device structures. ${ }^{1-4}$ An important novel optoelectronic device structure recently proposed is the light modulator based on the quantum well in the presence of an electric field. ${ }^{5-7}$ A number of experimental groups are currently exploring optical properties of $\mathrm{AlGaAs} / \mathrm{GaAs}$ quantum wells in the presence of an electric field. ${ }^{6-8}$ The interest in this structure arises due to the possibility of shifting the energy of the radiation emitted from the structure as well as strong quenching of photoluminescence (PL) at high electric fields.

Although a number of experimental results have been reported on $P \mathbb{L}$ under electric field in quantum wells, the theoretical understanding of this phenomenon is far from clear. A theoretical work based upon the variational principle has been reported in literature, but it appears to have difficulty in understanding the PL quenching at high fields. ${ }^{9}$ In this letter we use an approach based on Monte Carlo techniques to calculate the electron and hole quasibound levels in a quantum well. This approach does not depend upon a judicious choice for the form of the wave function (as the conventional variation approach does) since the Monte Carlo method scans all possible wave functions and selects the one with the lowest energy. At low fields we find good agreement between the results obtained by this approach and the one reported in literature by using the conventional variational approach, ${ }^{9}$ but at high fields there are significant differences. In particular, a much higher PL quenching is predicted by our calculations due to the high tunneling rates for the carriers. We also find that quasibound levels become unbound at a much lower electric field. We attribute these differences to the fact that in conventional variational approach an improper choice of the form of the wave function can lead to unreliable results. Recently calculations based on numerical integration of Schrödinger equation have been presented for the same problem, ${ }^{10}$ and we find that the Monte Carlo results agree well with these results.

According to the variational principle, the ground state energy $E_{0}$ corresponding to a Hamiltonian $H$ is determined from the condition

$$
E_{0}=\min \int \psi^{*} H \psi d r
$$

where

$$
H \psi=\left(\frac{-\hbar^{2}}{2 m} \nabla^{2}+V(r)\right) \psi(r)=E \psi(r)
$$

and

$$
\int|\psi|^{2} d r=1
$$

In principle, the approach requires one to consider all possible waveforms and select the one which gives the minimum energy. However, in practice one considers a well-defined waveform (based usually on physical intuition) with some parameters which are then varied to obtain the minimum energy for that waveform.

In this paper we will describe a method based on Monte Carlo techniques which finds a solution to the set of Eq. (1)(3), but is not limited to any special choice of the wave function. We will apply this technique to bound and quasibound electron-hole levels in a quantum well in presence of an electric field.

\section{THE MONTE CARLO METHOD}

The Monte Carlo method has been widely used in the areas of charge transport, ${ }^{11}$ crystal growth, ${ }^{12}$ solution of quantum mechanical problems, ${ }^{13}$ etc. To solve the set of Eqs. (1) $-(3)$ the following steps are performed:

(i) An arbitrary normalized wave function is chosen inside a fixed volume (see discussion on quasibound states).

(ii) The energy associated with this wave function is calculated using Eq. (1). The kinetic energy and potential energy of the particle are calculated for any given mass (which may vary with position) and potential $V(x)$.

(iii) Using a random number of small change is made in the wave function at a random point $x_{i}$.

(iv) The new wave function is normalized and its energy is recalculated.

(v) The new wave function is retained if (a) the energy of the new wave function is lower than that of the initial one or (b) a random number $R$ [called from a uniform 0 to 1 distribution is less than where $\delta$ is a small number 
$\left.\left(\sim 10^{-3}\right)\right]$. The case $b$ is allowed to ensure that the wave function is able to reach a true energy minimum.

The steps (iii) $-(v)$ are repeated until convergence is obtained. It is important to realize that the final solution is independent of the starting wave function although a judicious starting wave function can lower the number of iterations required to reach a stable solution. The above method is obviously not unique and other procedures can also be used, but the final results are quite independent of the methods.

\section{QUASIBOUND STATES}

The practical computer aspects of going through the steps (i)-(v) above require that the physical space over which the wave function extends is finite. A square quantum well may be defined by

$$
\begin{aligned}
V(x) & =0 \text { if } x_{0} \leqslant x \leqslant x_{0}+W \\
& =V_{0} \text { if } x<x_{0} \text { or } x>x_{0}+W .
\end{aligned}
$$

The space over which the bound state extends is typically $\sim W$ so that in the calculations one keeps information of distances a few times $W$. In case an electric field $E$ is applied to the quantum well, one has an additional potential given by

$$
\widetilde{V}(x)=e E x \text {. }
$$

In this case, the subband levels in the quantum well given by Eq. (4) can only be quasibound since for large enough $x$ away from the well the free electron (hole) states are lower (higher) in energy than the subband levels. Since the Monte Carlo method described earlier will always find the wave function with the lowest energy, it is clear that the wave function will depend on the size of the system considered. To find the true quasibound state we exploit the fact that a quasibound state is confined near the well with very small probability away from the well. The following technique is used. An arbitrary potential is superimposed on the potentials defined by Eqs. (4) and (5). This arbitrary potential is defined by

$$
\begin{aligned}
& V_{A}=V_{1} \quad x \leqslant x_{0}-L \text { or } x \geqslant x_{0}+W+L \text {, } \\
& =0 \quad \text { otherwise }
\end{aligned}
$$

where we choose $V_{1}>V_{0}$. The distance $L$ is gradually increased from 0 to higher values and the Monte Carlo approach described earlier is utilized to solve for the lowestenergy wave function. As the distance $L$ is increased one expects that the ground-state wave function will show one of the two schematic behaviors shown in Fig. 1 . In case of a true quasibound state, the energy associated with the wave function (as well as other properties such as shapes, etc.) stabilizes at a value of $L=L c_{1}$ and remains essentially invariant till $L=L c_{2}$ after which the energy rapidly changes. In case there is no quasibound state, the energy (and other properties) associated with the lowest-energy wave function changes uniformly as $L$ is changed. The physical argument behind this criteria is straightforward. If the ground state is a true quasibound state, it will be confined primarily near the quantum well region. $L c_{1}$ represents the distance up to which the wave function extends (of course an extremely

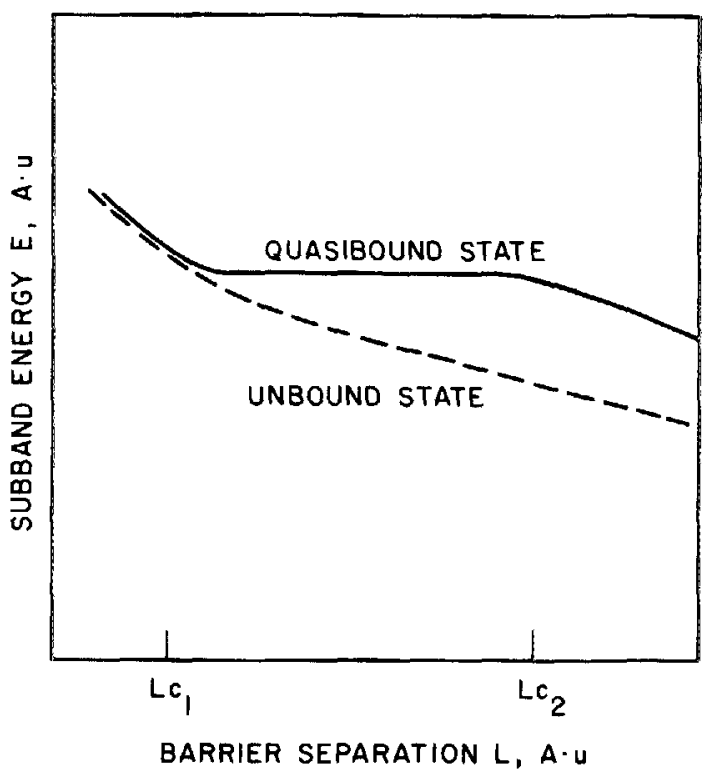

FIG. 1. Signatures of a quasibound (solid line) and unbound (dashed line) state as the parameter $L$ of the artificial barrier discussed in the text is increased. $E(L)$ is the lowest energy associated with the wave functions.

small fraction extends further into the barrier). Thus increase in $L$ does not significantly change the solution. However, beyond $L c_{2}$, the lowest energy states are the free band states. On the other hand, if the electric field is too high, one cannot talk about quasibound states [e.g., see Eq. (14) of Ref. 9J. This criteria is found to work extremely well for calculating the quasibound states as will be clear from the results presented below.

The method described in brief here is found to work very efficiently in determining quasibound levels, and does not suffer from the pitfalls of the choice of inaccurate waveform
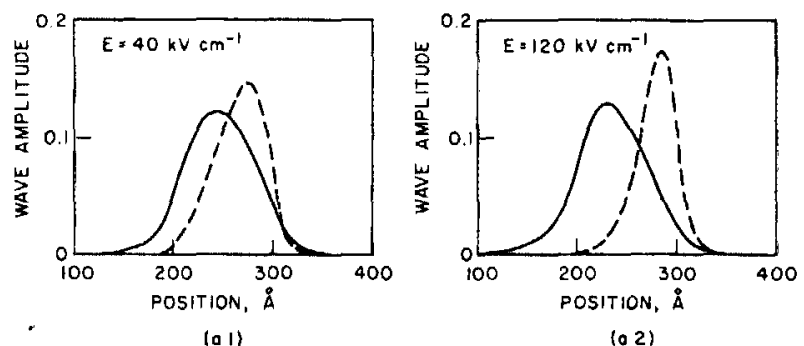

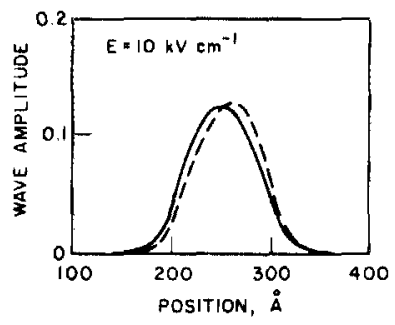

(b I)

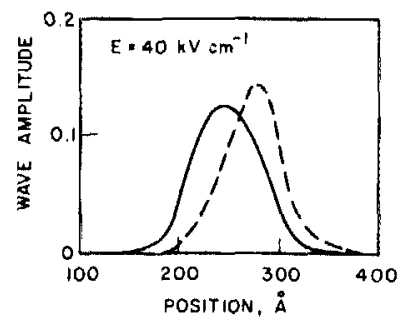

(b 2)
FIG. 2. Electron and hole wave functions in a $100-\AA \mathrm{Al}_{0.3} \mathrm{Ga}_{0,7} \mathrm{As} / \mathrm{GaAs}$ quantum well in the presence of an electric field of (a1 ) $40 \mathrm{kV} / \mathrm{cm}$ and (a2) $120 \mathrm{kV} / \mathrm{cm}$ with a band-edge discontinuity of $60: 40$ and (b1) $10 \mathrm{kV} / \mathrm{cm}$ and (b2) $40 \mathrm{kV} / \mathrm{cm}$ with a discontinuity of $85: 15$. The well is centered at $250 \AA$. 
in the conventional approach to the variational principle.

The above technique has been applied by us to determine the subband levels for the electron and hole in an $\mathrm{Al}_{0.3} \mathrm{Ga}_{0.7}$ As-GaAS- $\mathrm{Al}_{0.3} \mathrm{Ga}_{0.7}$ As quantum well. We have assumed a total band-gap discontinuity of $360 \mathrm{meV}$ across the interface which is distributed across the conduction and valence band in the ratio of $60: 40$ or $85: 15$. These two values have been chosen to display the effects of the discontinuity on the results. It is worth mentioning that the $85: 15$ discontinuity was suggested earlier by classic experiments of Dingle et al. ${ }^{14}$ but recently a much larger valence band discontinuity is being suggested. ${ }^{15}$ The effective masses of the electron and hole are taken to be $0.067 m_{0}$ and $0.45 m_{0}$, respectively, where $m_{0}$ is the free electron mass.

It is well known that if the quantum well is exposed to light, electron hole pairs are produced which form excitons and eventually recombine to emit photons with energy given by

$$
E_{p h}=E_{e}+E_{h}+E_{g}-E_{b} \text {, }
$$

where $E_{e}$ and $E_{h}$ are the electron and hole subband levels, $E_{g}$ is the band gap of the well material, and $E_{b}$ the exciton binding energy.

In the presence of an electric field, there is negligible change in $E_{g}$, but $E_{e}$ and $E_{h}$ change substantially. There is also comparatively small change in $E_{b}$ which is controlled by electron-hole overlap in the presence of the electric field (e.g., for a $100-\AA$ well a change of approximately $30 \%$ at an electric field of $80 \mathrm{kV} / \mathrm{cm}$ leading to a binding energy change of approximately $2 \mathrm{meV}$ ).

We have calculated the subband levels and the associated energies and wave functions which then allow us to calculate the tunneling probabilities for the electrons and holes. We note that the solutions presented here are based on a variational approach. In variational approaches, the eigenenergies are more reliable than the eigenfunctions. We have therefore used the WKB method to calculate the tunnel-

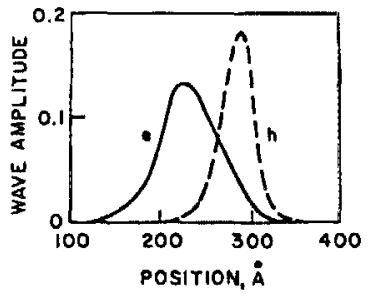

(o)

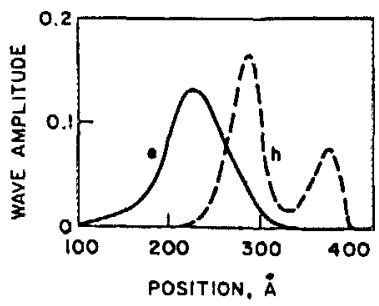

(c)

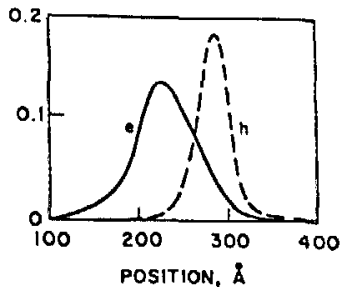

(b)

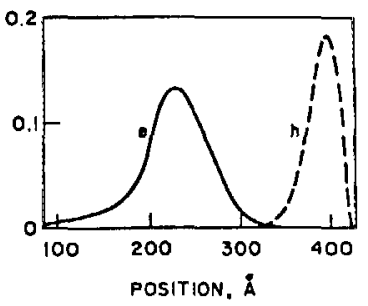

(d)
FIG. 3. Monte Carlo results for the electron and hole wave functions in presence of an electric field of $160 \mathrm{kV} \mathrm{cm}^{-1}$. At this high field, the hole state is not a quasibound state, since the hole wave function does not reach a stable state as the artificial barrier described in the text is removed from 60 to $120 \AA$ in steps of $20 \mathrm{~A}$.

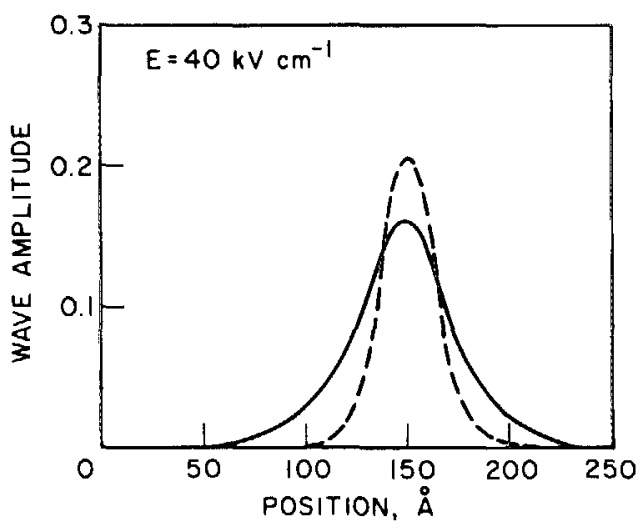

(a)

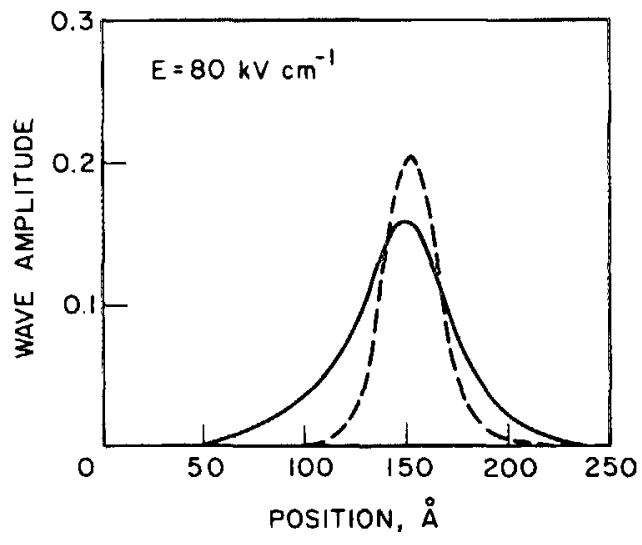

(0 2)

FIG. 4. Electron and hole wave functions in a $30-\AA \mathrm{Al}_{0.3} \mathrm{Ga}_{0.7} \mathrm{As} / \mathrm{GaAs}$ quantum well in the presence of an electric field of (a1) $40 \mathrm{kV} / \mathrm{cm}$ and (a2) $80 \mathrm{kV} / \mathrm{cm}$ with a band-edge discontinuity of $60: 40$. The well is centered at $150 \AA$ A.

ing rates for the electrons and the holes. Athough in principle the WKB method is an approximation, it has proven to be quite accurate in many tunneling related calculations. Of course, any fine structure in the tunneling rates is lost when the WKB approach is used.

The tunneling probability of a particle in a quasibound state with energy $E$ is given by the WKB expression ${ }^{16}$

$$
E_{p h}=\exp -\frac{1}{\hbar} \int_{x 1}^{x 2} \sqrt{8 m[V(x)-E]} d x,
$$

with an attempt frequency given by

$$
v=V / 2 L \text {, }
$$

where $V$ is the particle velocity calculated from the kinetic energy and $L$ is the spread of the wave function.

The results from our calculations are shown in Figs. 26. In Fig. 2, we show the electron and hole wave functions in a 100-A quantum well for discontinuities of $60: 40$ (case $a$ ) and 85:15 (case b). The electric fields are (a1) $E=40 \mathrm{kV} /$ $\mathrm{cm}$, (a2) $120 \mathrm{kV} / \mathrm{cm}$ (for case a) and (b1) $10 \mathrm{kV} / \mathrm{cm}$, (b2) $40 \mathrm{kV} / \mathrm{cm}$ (for case b).

In Fig. 3 we show the case when the applied electric field is $160 \mathrm{kV} \mathrm{cm}^{-1}$ and discontinuity is $60: 40$. In this case we show the wave functions calculated with $V_{1}$ in Eq. (6) cho- 


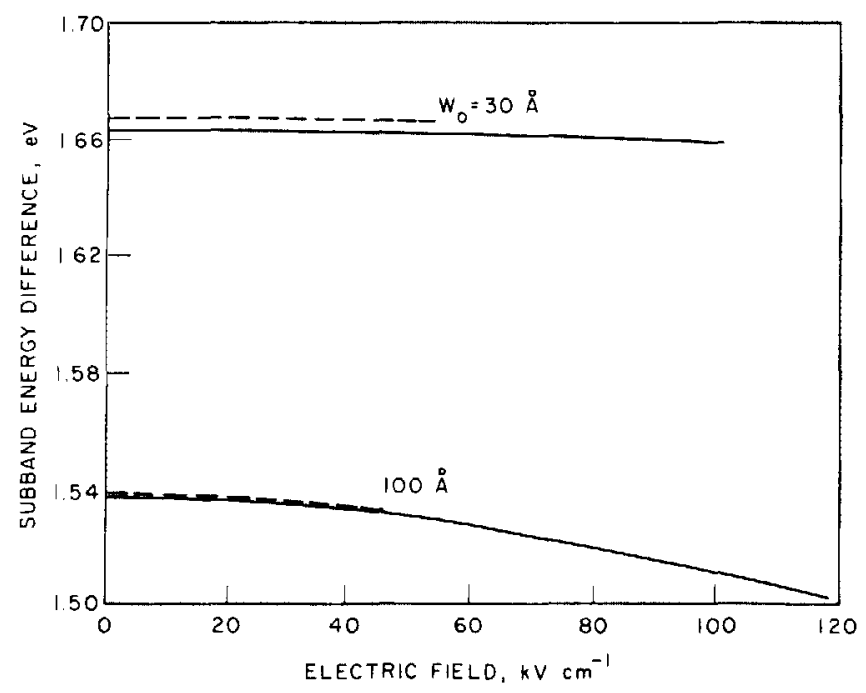

FIG. 5. Variation of the electron-hole subband level difference in the presence of an electric field for a 30 - and 100 - quantum well. The band-edge discontinuities used are 60:40 (solid line) and 85:15 (dashed line).

sen to be $1000 \mathrm{meV}$ for the electrons and for holes (the results are independent of the choice of $V_{1}$ as long as $V_{1}>V_{0}$ ); $V_{0}=216 \mathrm{meV}$ for electrons and $144 \mathrm{meV}$ for holes). The $L$ defined by Eq. (6) changes from 60 to $120 \AA$ in steps of $20 \AA$ for the four cases shown. It is clear that for this electric field, the hole wave function is not a quasibound state, but an unbound state. This is consistent with the following physical observation. The hole wave function penetrates $\sim 50 \AA$ beyond the barrier at $x=x_{0}+W_{0}$. At $E=160 \mathrm{kV} \mathrm{cm}^{-1}$ the shift in the band position over $50 \AA$ is $80 \mathrm{meV}$. Since the difference between the hole subband and the barrier at $x=w_{0}$ is also $\sim 80 \mathrm{meV}$, at such high fields, the wave function becomes unbound. Similarly for an 85:15 discontinuity, the hole level becomes unbound at $50 \mathrm{kV} \mathrm{cm}{ }^{-1}$. Note that this is in marked contrast with the variational calculation of Bastard et al. ${ }^{9}$ which suggests that the quasibound state continues to exist even at twice the electric fields mentioned above. We believe this difference arises because the latter authors force the variational wave function to have an exponential decay even at very high fields.

We have also obtained results for the electron and hole levels for the 30-A quantum well as shown in Fig. 4. The results are for a 60:40 discontinuity and an electric field of (a1) $40 \mathrm{kV} / \mathrm{cm}$ and (a2) $80 \mathrm{kV} / \mathrm{cm}$. It is important to note that due to the much larger spread of the electron wave function, the electron state becomes unbound for the 60:40 discontinuity at $90 \mathrm{kV} / \mathrm{cm}$. However, for the $85: 15$ discontinuity, the hole state becomes unbound at $50 \mathrm{kV} / \mathrm{cm}$ due to the small barrier seen by the hole.

In Fig. 5, we show the variation of the subband energy difference as a function of electric field for the 100 - and $30-\AA$ quantum wells for the two cases of the discontinuity: (a) 60:40 (solid line) and (b) 85:15 (dashed line). We find that for the $100-\AA$ well, it is possible to change the subband energy difference considerably before the system becomes unbound. For the $30-\AA$ well, there is a very small shift with the electric field. Figure 3 also shows an insignificant depen-

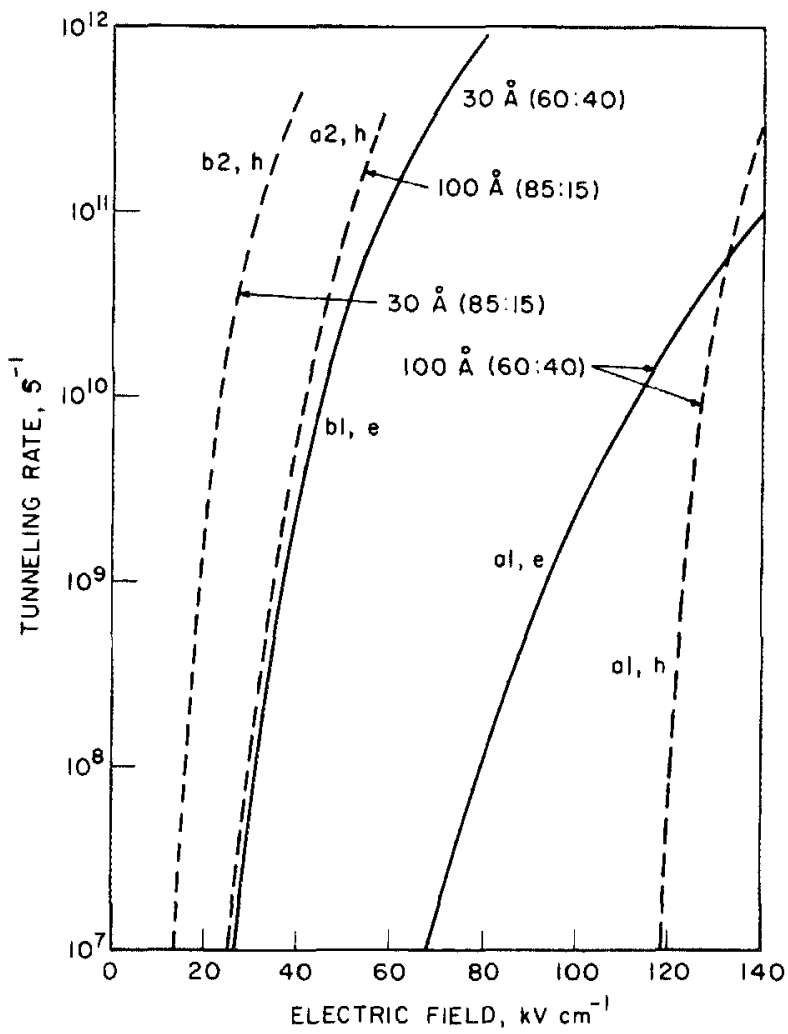

FIG. 6. Tunneling rates for electrons (solid line) and holes (dashed line) from 100 - and $30-\AA \mathrm{Al}_{0.3} \mathrm{Ga}_{0.7} \mathrm{As} / \mathrm{GaAs}$ quantum wells. The various cases are (a1) discontinuity $=60: 40$, well size $=100 \AA$; (a2) discontin uity $=85: 15$, well size $=100 \AA$; (bl) discontinuity $=60: 40$, well size $=30$ $\AA$; (b2) discontinuity $=85: 15$, well size $=30 \AA$.

dence of the subband energy difference on the discontinuity assumed.

Finally in Fig. 6, we show the tunneling rates for the electrons and holes for the cases discussed earlier. A study of the effect of these tunneling rates on optical quenching will be discussed elsewhere, but we point out here that these rates must be compared to $\sim 10^{9} \mathrm{~s}^{-1}$ for exciton collapse. When the tunneling times $\left(\tau_{\text {tun }}=1 / T v\right)$ become comparable to the exciton collapse time, the intensity of the emission light from the structure will decrease rapidly. We point out that if the electron and holes are created above the quantum well effective band gap, the tunneling rate will be even higher due to the higher electron or hole kinetic energies. It is important to note the tremendous sensitivity of the tunneling rates to be discontinuities at the conduction and valence bands. We have only reported the rates up to fields where both the electron and hole states are quasibound.

The results presented here agree rather well with the trends reported in the literature..$^{5-8}$ For example, the shift in emission energy with increased applied bias and the PL quenching appear to be consistent with our calculations. However, at this stage a detailed comparison is not advisable due to the rather sketchy information available on electric field versus emission energy and intensity.

In summary, we have presented a new formalism for tackling the problem of quasibound wells in an electric field which is quite different from the existing results based on the 
variation principle. We believe that the Monte Carlo approach presented here will have useful application in understanding electro-optical devices, since it can be applied to arbitrary-shaped quantum wells.

${ }^{1}$ C. E. C. Wood, in Physics of Thin Films, edited by George Haas and M. H. Francombe (Academic, New York, 1980), Vol. 11, p. 35.

${ }^{2}$ A. Y. Cho, Thin Solid Films 100, 291 (183).

${ }^{3}$ J. R. Arthur, Surf. Sci. 43, 449 (1974).

${ }^{4}$ See, for example, articles published in Praceedings of the International Conference on Metalorganic Vapor Phase Epitaxy, edited by J. F. Bonfils, S. J. Irvine, and J, B. Mullin (North-Holland, Amsterdam, 1981).

${ }^{\text {S}}$ E. E. Mendez, G. Bastard, L. L. Chang, L. Esaki, H. Morkoc, and R. Fischer, Phys. Rev. B 26, 7101 (1982).

${ }^{6}$ T. H. Wood, C. A. Burrus, D. A. B. Miller, D. S. Chemla, T. C. Dammen, A. C. Gossard, and W. Wiegman, Appl. Phys. Lett. 44, 16 (1984).

${ }^{7}$ W. D. Goodhue, B. E. Burke, K. B. Nichols, G. M. Metze, and G. D. Johnson, presented at the Sixth MBE Workshop, Aug. 14-16, 1985, Minneapolis, MN.
${ }^{8}$ P. W. Yu, K. K. Bajaj, D. C. Reynolds, C. W. Litton, J. Singh, R. Fischer, and $\mathbf{H}$. Morkoc (unpublished).

${ }^{9}$ G. Bastard, E. E. Mendez, L. L. Chang, and L. Esaki, Phys. Rev. B 28, 3241 (1983).

${ }^{10}$ D. A. B. Miller, D. S. Miller, D. S. Chemla, T. C. Damen, A. C. Gossard, W. Wiegmann, T. H. Wood, and C. A. Burrus, Phys. Rev. B 32, 1043 (1985).

${ }^{11}$ W. Fawcett, A. D. Boardman, and S. Swain, J. Phys. Chem. Solids 31, 1963 (1969).

${ }^{12}$ J. D. Weeks, G. H. Gilmer, and K. A. Jackson, J. Chem. Phys. 65, 712 (1976).

${ }^{13}$ R. K. Kalia, P. Vashishta, and M. A. Lee, Solid State Commun. 53, 1138 (1985).

${ }^{14} \mathrm{R}$. Dingle, in Festkorperprobleme (Advances in Solid State Physics), edited by H. J. Oveisser (Pergamon, Braunschweig, 1975), Vol. XV, p. 21.

${ }^{15}$ R. C. Miller, D. A. Kleinman, and A. C. Gossard, Phys. Rev. B 29, 7085 (1984).

${ }^{16}$ L. D. Landau and E. M. Lifshitz, Quantum Mechanics-Non-Relativistic Theory (Pergamon, New York, 1981), Chap. VII. 